\title{
Radiative corrections and Monte Carlo generators for physics at flavor factories
}

\author{
Guido Montagna ${ }^{1, a}$ \\ ${ }^{1}$ Dipartimento di Fisica, Università di Pavia and INFN, Sezione di Pavia, \\ Via A. Bassi 6, 27100, Pavia, Italy
}

\begin{abstract}
I review the state of the art of precision calculations and related Monte Carlo generators used in physics at flavor factories. The review describes the tools relevant for the measurement of the hadron production cross section (via radiative return, energy scan and in $\gamma \gamma$ scattering), luminosity monitoring, searches for new physics and physics of the $\tau$ lepton.
\end{abstract}

\section{Introduction}

The physics program of $e^{+} e^{-}$colliders with center of mass (c.m.) energy between about 1 and $10 \mathrm{GeV}$ (flavor factories) is broad and ranges from flavor physics to the measurement of the low-energy hadronic cross section, with its crucial implications for $g-2$ and the running of $\alpha$, to searches for QCD exotic states and studies of QCD in the non-perturbative regime. It also includes precision tests of the SM at low energies and new physics (NP) searches (dark photon, light Higgs bosons), as well as measurements related to the physics of the $\tau$ lepton.

The reason why radiative corrections (RC) and accurate Monte Carlo (MC) generators are involved in most of these studies stems from the fact that the large statistics accumulated by these experiments at the intensity frontier necessarily requires precision calculations for a large spectrum of measurements and the description of physics phenomena. Examples are the simulation of the contribution due to initial-state radiation (ISR) and the measurement with $1 \%$ precision or better of the hadronic cross section via the mechanism of radiative return; the modeling of final-state radiation (FSR) and bremsstrahlung in decays (for example, in the leptonic decay of the $\tau$ ); the measurement of the collider luminosity with per mille uncertainty and the control of the process of muon pair production in association with a photon as normalization of the hadronic cross section measurement via radiative return; and, finally, the simulation of QED processes, considered both as signals and backgrounds, depending on the measurement.

All these physics issues require that the precision calculations are made available in the form of MC generators, which are needed to allow for simulations and data-theory comparisons under complex selection criteria.
The theoretical methods used in this context mostly refer to the QED sector of the SM and include results at NLO in perturbation theory and, in some special cases, at NNLO. Also calculations addressing the resummation of large contributions due photon collinear emission are involved. QED resummation is performed according to different approaches, like collinear Structure Functions (SFs), Parton Shower (PS) or YFS exponentiation. In precision calculations for luminosity and QED processes, predictions based on the matching of NLO corrections with higher-order (h.o.) contributions are also available.

A working group (WG) on $\mathrm{RC}$ and $\mathrm{MC}$ for low energies was promoted in the middle of the 2000s, in order to stimulate and facilitate the collaboration between experimentalists and theorists working on physics at meson factories. It organizes two meetings per year (see http://www. Inf.infn.it/wg/sighad/) and led in 2010 to the publication of the review paper of Ref. [1], which stands as a reference in the field also today.

\section{Hadron production cross section}

At flavor factories, the hadronic cross section is measured via radiative return [2] (hadron production in association with an energetic ISR photon) and energy scan, as well as in $\gamma \gamma$ scattering.

In spite of the $\alpha$ reduction factor associated to photon radiation, the hadronic cross section via radiative return can be measured from threshold to the nominal c.m. energy with good precision (at the one per cent level or better), thanks to the large luminosity of flavor factories. In this context, the reference MC used by all the experiments as primary generator or to cross-check the accuracy of other tools is PHOKHARA [3-8]. It is the successor of EVA, the first MC developed for this kind of studies in Ref. [9] for the two pion + photon final state and in Ref. [10] for the 4 pions + photon signature, with a treat-

\footnotetext{
a e-mail: guido.montagna @ pv.infn.it
} 
ment of the ISR hard photon at LO supplemented by QED collinear SFs for the description of additional undetected radiation. ${ }^{1}$ Now PHOKHARA can be used to simulate various hadronic final states, both for measurements with explicitly tagged or untagged photons. The treatment of ISR is at NLO accuracy for the all the final states, with an estimated precision of $0.5 \%$, due to missing h.o. contributions beyond $O(\alpha)$ in the calculation of the radiator function. For two pions, two protons and $\mathrm{K}^{+} \mathrm{K}^{-}$final states also FSR is treated at NLO, using the point-like approximation. In the latest version of PHOKHARA, the complete set of NLO corrections is available for $\mu^{+} \mu^{-} \gamma$ production [14], a process often used as normalization in the measurement of the hadron $+\gamma$ production cross section. The recent KLOE and BESIII measurements of the $\pi^{+} \pi^{-} \gamma$ cross section, of primary interest for the calculation of the leading order hadronic contribution to the $g-2$, were based on PHOKHARA, whereas in the analyses by BaBar the code AfkQED is adopted.

Concerning the measurements of the hadronic cross section with the method of energy scan, the situation of the MC generators is less established and more varied. What is available in the literature is MCGPJ [15], a generator developed by a Dubna-Novosibirsk collaboration and used at VEPP collider. It can presently simulates the $\pi \pi$ and $K K$ production channels but the implementation of new multi-hadron final states is in progress. The code includes the complete set of NLO QED corrections plus h.o. contributions treated in terms of collinear SFs and makes use of Scalar QED for the calculation of FSR corrections. Therefore, this generator is quite accurate and has an estimated accuracy of $0.2 \%$. A further available code is PHOKHARA 8.0 [16], that can be used to simulate the hadron cross section measured in energy scan for the same channels as in the PHOKHARA version for radiative return. Here, ISR is treated at NNLO accuracy, but without the contribution of multiple photon emission and FSR. The predictions of PHOKHARA 8.0 are in very good agreement, at a few per mille level, with those of MCGPJ for the two pion final state and with those of a benchmark $\mathrm{MC}, \mathrm{KKMC}[17]^{2}$, for muon pair production. Another interesting, ongoing project is carlomat 3.0 [19], which is an automatic tool for the generation of the scattering amplitudes and cross sections related to complex topologies given by hadrons + photons + leptons. In carlomat 3.0 the calculations are performed using the effective vertices of the Resonance Chiral Lagrangian $(R \chi L)$ Theory or Hidden Symmetry model and, for the time being, RC are not included.

${ }^{1}$ The EVA approach is followed by BaBar collaboration in their analysis of hadron production via radiative return processes, using their own, not public event generator AfkQED. In the latter the muon final state is simulated independently according to the formulae of Ref. [11]. The usage of AfkQED in BaBar is supplemented by PHOTOS [12, 13] for the description of additional undetected FSR.

${ }^{2} \mathrm{KKMC}$ is a precise $\mathrm{MC}$ tool for the simulation of several $2 \rightarrow 2$ processes [18]. It is based on a generalization of the YFS exponentiation approach (coherent exclusive exponentiation) and is used at flavor factories in various studies requiring simulations of lepton-pair production or QED radiative processes.
For $\gamma \gamma$ physics, of special interest for the measurements of the photon-meson transition form factors and tests of QCD, the various codes used till now by the experiments are all characterized by the common feature of using the Equivalent Photon Approximation (EPA) for the cross section calculation. A more precise generator, beyond the EPA, is EKHARA [20], which includes exact formulae and exact kinematics for both $s$ - and $t$-channel amplitudes and their interference. It allows to obtain predictions for $e^{+} e^{-} \pi^{+} \pi^{-}$production, as well as for $e^{+} e^{-} \pi^{0}, e^{+} e^{-} \eta / \eta^{\prime}$ production, using different models for the photon-meson transition form factors. The implementation in EKHARA of the dominant QED corrections is under way and will available in future releases.

\section{Luminosity measurement}

The area where a particular progress occurred, both in the calculation of RC and realization of MC codes, is that related to the luminosity measurement. Indeed, at flavor factories, the luminosity is measured with very good precision, typically with an uncertainty between a few per mille and one per cent, by using the ratio between the observed number of events of a given reference process and the corresponding theoretical cross section through the relation $\mathcal{L}=N_{\text {obs }} / \sigma_{\text {theory }}$. To maintain as small as possible the total luminosity uncertainty, the best reference process is largeangle Bhabha (LABH) scattering, measured in the same detectors used for the measurement of the hadronic cross section, with similar acceptance conditions. Two photon production is also used a cross check or to obtain the luminosity as an average of the measurements obtained with the two processes. In the luminosity measurement, typically two independent generators are used to avoid loss of precision or introduce a bias in the measurement itself.

Table 1. The MC generators used in luminosity measurements.

\begin{tabular}{llll}
\hline Generator & Processes & Theory & Accuracy \\
\hline \hline BabaYaga 3.5 & $e^{+} e^{-}, \gamma \gamma, \mu^{+} \mu^{-}$ & QED PS & $0.5 \%$ \\
\hline BabaYaga@NLO & $e^{+} e^{-}, \gamma \gamma, \mu^{+} \mu^{-}$ & $O(\alpha)+$ QED PS & $0.1 \%$ \\
\hline BHWIDE & $e^{+} e^{-}$ & $O(\alpha)$ YFS & $0.1 \%$ \\
\hline MCGPJ & $e^{+} e^{-}, \gamma \gamma, \mu^{+} \mu^{-}$ & $O(\alpha)+$ SFs & $0.2 \%$ \\
\hline
\end{tabular}

The generators used to monitor the luminosity at meson factories are summarized in Table 1. The reference code adopted by most of the experimental collaborations is the generator BabaYaga [21-23]. In its most precise version, BabaYaga@NLO, the generator includes the exact NLO corrections matched to a QED PS for the simulation of exclusive multiple photon emission and has an estimated accuracy of $0.1 \%$. Other programs used at flavor factories for simulations of the LABH process with a formulation and precision similar to that of BabaYaga@NLO are BHWIDE [24], developed during the LEP time, and MCGPJ, which, in addition to hadronic final states, provides predictions for lepton pair and two-photon [25] production.

Because of the precision requirements, a relevant question in the context of luminosity measurements is related 
to the accuracy of the theoretical calculations and corresponding codes. As a consequence of the formulation underlying the most precise generators, featuring the matching of NLO corrections with QED exponentiation in factorized form, the bulk of the most important sub-leading $O\left(\alpha^{2}\right)$ corrections, i.e. $O\left(\alpha^{2} L\right)$ photonic corrections ( $L$ being the large collinear logarithm), is effectively incorporated [26]. It follows that the main uncertainties affecting the theoretical precision of the luminosity generators come from two sources. The first one is related to the hadronic contribution to the vacuum polarization, which is treated using dispersion relations and is therefore parametric, driven the experimental uncertainty of the hadron cross section measurements. The second source of uncertainty is purely perturbative, due to the incomplete inclusion of QED corrections at NNLO. Fortunately, all the pieces of the Bhabha cross section at NNLO in QED have been computed over the last decade or so (see e.g. Ref. [1]) and therefore these calculations represent an important benchmark to assess the theoretical precision of the luminosity MC tools.

Within the WG on RC and MC for low energies, a particular effort was devoted to reach a reliable estimate of the theoretical uncertainty of the luminosity cross section calculation. Following similar work done during the LEP workshops in the 1990s, detailed comparisons between the predictions of the available NNLO calculations and the corresponding approximations present in BabaYaga@NLO led to the estimate of the total theoretical uncertainty summarized in Table 2 . It quotes the official WG evaluation updated by the conclusions of the work on leptonic and hadronic pair corrections of Ref. [27] and by a less conservative estimate of the one-loop corrections to hard bremsstrahlung according to Ref. [28].

\begin{tabular}{llll}
\hline Source of unc. (\%) & $1-2 \mathrm{GeV}$ & BESIII & BaBar/Belle \\
\hline \hline$\left|\delta_{\mathrm{VP}}\right|[$ Jegerlehner] & - & 0.01 & 0.03 \\
$\left|\delta_{\mathrm{VP}}\right|[\mathrm{HMNT}]$ & 0.02 & 0.01 & 0.02 \\
\hline$\left|\delta_{\text {photonic }}^{\alpha^{2}}\right|$ & 0.02 & 0.02 & 0.02 \\
$\left|\delta_{\text {pairs }}^{\alpha^{2}}\right|$ & 0.03 & 0.02 & $0.03 \div 0.07$ \\
$\left|\delta_{\mathrm{SV}, \mathrm{H}}^{\alpha^{2}}\right|$ & $0.05 / 0.03$ & $0.05 / 0.03$ & $0.05 / 0.03$ \\
$\left|\delta_{\mathrm{HH}}^{\alpha^{2}}\right|$ & - & - & - \\
\hline \hline$\left|\delta_{\text {total }}\right|$ & $0.07 / 0.05$ & $0.06 / 0.04$ & $\sim 0.07 \div 0.09$ \\
\hline
\end{tabular}

Table 2. The total theoretical uncertainty of the LABH scattering cross section at flavor factories.

Note that the estimate of the total theoretical uncertainty in the luminosity measurement at flavor factories, between $0.05 \%$ and $0.1 \%$, is comparable to that achieved at LEP in the 1990s. However, it slightly deteriorates in the proximity of the very narrow resonances because of the uncertainty induced by the hadronic contribution to the vacuum polarization.

\section{Search for New Physics and $\tau$-lepton physics}

The generators involved in the search for NP and physics of the $\tau$ lepton concern the simulation of QED processes, the modeling of $\tau$ decays and the description of bremsstrahlung in $\tau$ decays.

Concerning NP searches, a particular attention was paid over the last few years in the search for a massive dark photon weakly coupled to the SM particles as a possible solution of the dark matter puzzle. One the best signals at meson factories is represented by the associated production of a dark photon and an energetic photon, with subsequent decay of the dark photon into electron or muon pairs. The signature consists of very narrow bumps in the lepton invariant mass spectrum, because of the very small coupling of the new particle to the leptons, over the continuum given by the QED background processes $e^{+} e^{-} \rightarrow e^{+} e^{-} \gamma, \mu^{+} \mu^{-} \gamma$. In this framework, an upgrade of BabaYaga [29] is used by KLOE for the simulation of both signal and backgrounds, whereas BaBar makes use of MadGraph/MadEvent [30] for signal simulations and of the tandem of generators BHWIDE/KKMC for the study of the QED radiative processes.

As far as physics of the $\tau$ lepton is concerned, the measurements giving access to the $\tau$ mass and related to $\tau$ pair production at threshold involve MC programs developed during the LEP era. The reference code used by BaBar, Belle and BESIII for the calculation of the $e^{+} e^{-} \rightarrow \tau^{+} \tau^{-}$ cross section is KKMC, but in some measurements also the older KORALB [31] generator is considered. For the measurements and physics studies related to $\tau$ decays, the reference tool is TAUOLA [32-34], which is a wellestablished program with a long tradition from LEP. Its usage is often accompanied by the process independent tool PHOTOS for the simulation of bremsstrahlung in $\tau$ leptonic decays. A recent interesting progress in TAUOLA has been the implementation of the hadronic currents using the $R \chi L$ theory, which supersedes previous theoretical parameterizations [35].

\section{Summary}

A wide spectrum of measurements and physics studies at flavor factories needs for precision calculations, including all the relevant RC, encoded into accurate and flexible MC generators. They are primarily required for simulations in the measurement of the hadron production cross section (via radiative return, energy scan and in $\gamma \gamma$ scattering), precise luminosity monitoring and control of the $\mu^{+} \mu^{-} \gamma$ normalization process. They are also involved in searches for NP and physics of the $\tau$ lepton.

Over the last decade or so, a significant progress occurred in theoretical predictions and related codes for the measurement of the hadronic cross section via radiative return and high-precision luminosity determination. The latter, in particular, took advantage of the computation of the full set of NNLO corrections to Bhabha process in QED. As a consequence, the accuracy of the presently available 
predictions for the luminosity cross section at flavor factories is at the sub-per mille level and robust.

More generally, theoretical results and generators used in simulations for luminosity, QED processes, NP searches and $\tau$ physics greatly benefited from LEP experience.

Further progress is expected in generators for the measurement of the hadron production cross section with energy scan and in $\gamma \gamma$ scattering processes. Presumably, new results will also come in the assessment of the theoretical uncertainty of the luminosity cross section as due to the hadronic contribution to the vacuum polarization close to $\psi / \Upsilon$ 's resonance and one-loop corrections to hard bremsstrahlung in Bhabha scattering.

\section{Acknowledgments}

I am grateful to M. Passera and G. Venanzoni for their kind invitation to a very interesting workshop in a very beautiful place. I wish to thank C.M. Carloni Calame, O. Nicrosini and F. Piccinini for the collaboration which led to the results on the luminosity cross section calculations here summarized. Useful correspondence with H. Czyż on PHOKHARA is gratefully acknowledged. I also want to acknowledge the continuous effort by H. Czyż and G. Venanzoni in the organization of the activity of the WG on $\mathrm{RC}$ and $\mathrm{MC}$ for low energies which constitutes the forum for the results here presented.

\section{References}

[1] S. Actis et al. [Working Group on Radiative Corrections and Monte Carlo Generators for Low Energies Collaboration], Eur. Phys. J. C 66, 585 (2010), arXiv:0912.0749 [hep-ph].

[2] A. B. Arbuzov, E. A. Kuraev, N. P. Merenkov and L. Trentadue, JHEP 9812, 009 (1998), hepph/9804430.

[3] G. Rodrigo, H. Czyz, J. H. Kuhn and M. Szopa, Eur. Phys. J. C 24, 71 (2002), hep-ph/0112184.

[4] H. Czyz, A. Grzelinska, J. H. Kuhn and G. Rodrigo, Eur. Phys. J. C 27, 563 (2003), hep-ph/0212225.

[5] H. Czyz, A. Grzelinska, J. H. Kuhn and G. Rodrigo, Eur. Phys. J. C 33, 333 (2004), hep-ph/0308312.

[6] H. Czyz, J. H. Kuhn, E. Nowak and G. Rodrigo, Eur. Phys. J. C 35, 527 (2004), hep-ph/0403062.

[7] H. Czyz, A. Grzelinska, J. H. Kuhn and G. Rodrigo, Eur. Phys. J. C 47, 617 (2006), hep-ph/0512180.

[8] H. Czyz, A. Grzelinska and J. H. Kuhn, Phys. Rev. D 81, 094014 (2010), arXiv:1002.0279 [hep-ph].

[9] S. Binner, J. H. Kuhn and K. Melnikov, Phys. Lett. B 459, 279 (1999), hep-ph/9902399.

[10] H. Czyz and J. H. Kuhn, Eur. Phys. J. C 18, 497 (2001), hep-ph/0008262.

[11] A. B. Arbuzov, G. V. Fedotovich, E. A. Kuraev, N. P. Merenkov, V. D. Rushai and L. Trentadue, JHEP 9710, 001 (1997), hep-ph/9702262.
[12] E. Barberio, B. van Eijk and Z. Was, Comput. Phys. Commun. 66, 115 (1991).

[13] E. Barberio and Z. Was, Comput. Phys. Commun. 79, 291 (1994).

[14] F. Campanario, H. Czyz, J. Gluza, M. Gunia, T. Riemann, G. Rodrigo and V. Yundin, JHEP 1402, 114 (2014), arXiv:1312.3610 [hep-ph].

[15] A. B. Arbuzov, G. V. Fedotovich, F. V. Ignatov, E. A. Kuraev and A. L. Sibidanov, Eur. Phys. J. C 46, 689 (2006), hep-ph/0504233.

[16] H. Czyz, M. Gunia and J. H. Kuhn, JHEP 1308 (2013) 110, arXiv:1306.1985 [hep-ph].

[17] S. Jadach, B. F. L. Ward and Z. Was, Phys. Rev. D 63, 113009 (2001), hep-ph/0006359.

[18] S. Jadach, B. F. L. Ward and Z. Was, Phys. Rev. D 88 (2013) 11, 114022, arXiv:1307.4037 [hep-ph].

[19] K. Kolodziej, arXiv:1504.05915 [hep-ph].

[20] H. Czyz and S. Ivashyn, Comput. Phys. Commun. 182, 1338 (2011), arXiv:1009.1881 [hep-ph].

[21] C. M. Carloni Calame, C. Lunardini, G. Montagna, O. Nicrosini and F. Piccinini, Nucl. Phys. B 584, 459 (2000), hep-ph/0003268.

[22] G. Balossini, C. M. Carloni Calame, G. Montagna, O. Nicrosini and F. Piccinini, Nucl. Phys. B 758, 227 (2006), hep-ph/0607181.

[23] G. Balossini, C. Bignamini, C. M. C. Calame, G. Montagna, O. Nicrosini and F. Piccinini, Phys. Lett. B 663, 209 (2008), arXiv:0801.3360 [hep-ph].

[24] S. Jadach, W. Placzek and B. F. L. Ward, Phys. Lett. B 390, 298 (1997), hep-ph/9608412.

[25] S. I. Eidelman, G. V. Fedotovich, E. A. Kuraev and A. L. Sibidanov, Eur. Phys. J. C 71 (2011) 1597, arXiv:1009.3390 [hep-ex].

[26] G. Montagna, O. Nicrosini and F. Piccinini, Phys. Lett. B 385 (1996) 348, hep-ph/9605252.

[27] C. Carloni Calame et al., JHEP 1107, 126 (2011), arXiv:1106.3178 [hep-ph].

[28] B. F. L. Ward, S. Jadach, M. Melles and S. A. Yost, Phys. Lett. B 450, 262 (1999), hep-ph/9811245.

[29] L. Barze, G. Balossini, C. Bignamini, C. M. C. Calame, G. Montagna, O. Nicrosini and F. Piccinini, Eur. Phys. J. C 71, 1680 (2011), arXiv:1007.4984 [hep-ph].

[30] J. Alwall et al., JHEP 0709, 028 (2007), arXiv:0706.2334 [hep-ph].

[31] S. Jadach and Z. Was, Comput. Phys. Commun. 36, 191 (1985).

[32] S. Jadach, J. H. Kuhn and Z. Was, Comput. Phys. Commun. 64, 275 (1990).

[33] M. Jezabek, Z. Was, S. Jadach and J. H. Kuhn, Comput. Phys. Commun. 70, 69 (1992).

[34] S. Jadach, Z. Was, R. Decker and J. H. Kuhn, Comput. Phys. Commun. 76, 361 (1993).

[35] I. M. Nugent, T. Przedzinski, P. Roig, O. Shekhovtsova and Z. Was, Phys. Rev. D 88 9, 093012 (2013), arXiv:1310.1053 [hep-ph]. 